\title{
Joint infection: a forbidden diagnosis?
}

\author{
Peter Angele ${ }^{1,2} \cdot$ Andreas Voss $^{1,2}$ \\ Published online: 4 September 2021 \\ (c) European Society of Sports Traumatology, Knee Surgery, Arthroscopy (ESSKA) 2021
}

Nowadays, orthopedic surgeons can use a huge variety of newly emerging surgical techniques for joint therapy. These techniques open the perspective of having complete and short patient recovery with "restitutio ad integrum" following injury. Nonetheless, postoperative joint infection is a rare but severe complication that can easily destroy the dreams of surgeon and patients to achieve full joint recovery after treatment. Specifically, chronic joint infections that persist over weeks can lead to expensive treatment costs and result in reduced clinical function in the long term.

Therefore, our ultimate goal in every surgical treatment should be to achieve our desired treatment goal and to avoid joint infection. However, in case of a joint infection, we should recognize and treat the infection, as early and efficiently as possible.

\section{How can we avoid joint infection?}

Every surgical treatment, including intraarticular injections, should follow the rules of antisepsis, which was introduced by Lister in the 1870s and still remain the principles of surgical care [5]. Antisepsis targets the destruction of germs on the surgical field, surgical materials and dressings on the hands of surgeons and equipment.

The ultimate goal of antisepsis should drive our clinical action every second, when we surgically treat patients, using the knowledge developed over hundreds of years, when surgeries were performed without sterilization. Antisepsis has only evolved in recent history, perhaps this historical fact, shows that biology is on our side and not against us.

Timing of surgery plays an important role to avoid infection. The patient with its injury has to be treated at the most

Peter Angele

angele@sporthopaedicum.de

1 Department of Trauma Surgery, University Medical Center Regensburg, Regensburg, Germany

2 Sporthopaedicum Regensburg, Regensburg, Germany optimal time point for the patient and not according to the surgeon's schedule or preference. The surgical profession has always been linked to myths and legends. The patient's hope has been in the surgeon's ability. Would these special abilities fill the hopeless situation with new hope? It was felt to be an almost magical moment, when the surgeons were able to save the apparently hopeless cases. Then, they were born, the "gods in white". However, our main driving force of action should be to help our patients in their difficult situation and not keeping these ancient habits.

Experience of the surgeon and the team help to perform fast and safe surgeries. Training of surgeons is necessary but for the sake of the patient, training is best performed with a short learning curve and low complication rates in centers, where the specific surgical procedure is frequently performed.

\section{How can we diagnose joint infection?}

Signs of infection, such as joint swelling, reddening, overheating, pain and limited range of motion are known since old Roman and Greek times. The difficulty in the diagnostic approach is to see the difference between a real postoperative septic arthritis and a postoperative hyperinflammation. The presentation in our daily practice of both can be very similar and the diagnosis may not be obvious and mentioned signs of a joint infection can be masked. Therefore, always be cautious, if the follow-up of a patient seems to be not normal.

The major difficulty is to diagnose an infection in his own patient cohort. The surgeon always tends to see infection only in patients from other surgeons. The misdiagnosis of his own infection complication is the major hurdle for diagnosis. Whenever, we only think about an infection, we should treat it as an infection. Fortunately, in this case, there are a number of laboratory and microbiological methods available to confirm an infection. 
Peter Angele

Andreas Voss

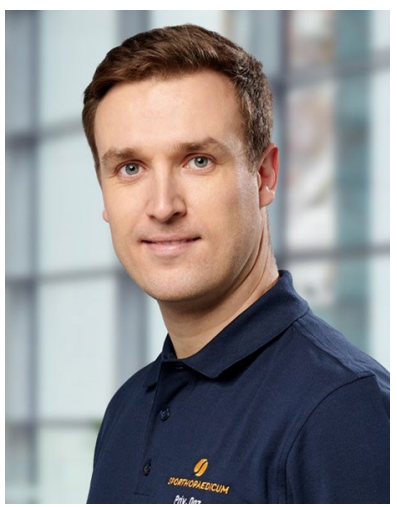

\section{How can we treat joint infection?}

The principles of joint infection treatment go back to Hippokrates, who claimed "Ubi pus ibi evaqua" which means that pus has to be removed by a surgical treatment. We know, that most antibiotics have good penetration into joint tissues but this is only helpful when the causative pathogen has been surgically reduced.
This view was not shared in the middle ages, as for many centuries the dogma amongst surgeons was that pus was needed and beneficial for healing. Also, Hippocrates commented on wounds and noticed that "if the pus is white, health will follow", but if it is "sanious and muddy, death will follow. This dogma mainly goes back to the Greek physician and philosopher, Galen of Pergamon, who claimed the phrase "laudable pus". An interesting article by Jeffrey A. Freiberg, who really went deep into the ancient times of wound healing and infection working up the myths of "laudable pus" [3]. Interestingly, Galen himself did not believe that pus was necessary for wound healing. He actually advocated crude medical therapies designed to dry wounds and decrease the amount of secretion. The possibly wrongly imposed statement comes from an assertion that in the case of abscesses, it is absolutely necessary that pus can drain off. Failure to drain can be fatal in the further course. This means that the absence of pus was interpreted as a possible cause of death and transferred to any wound healing.

Only with the work on hygiene, the studies on the theory of germs and antiseptic techniques by Ignaz Semmelweis[4], Louis Pasteur, and Joseph Lister in the nineteenth century the dogma "laudable pus " was gradually left.

\section{The century of antibiotics}

With the discovery of penicillin by Alexander Fleming in 1928, the concept of antimicrobial treatment was revolutionized and opened a completely new field of treatment 
options [1]. During the course of the twentieth century, more and more antibiotics have been discovered and another ground breaking new antibiotic was cephalosporin (by Abraham and Newton 1948), which is still one of the most used antibiotics in trauma and orthopedic surgery.

The use of antibiotics both in the medical field and animal foodstuff industry, alongside the breeding of modern livestock, nature has found a way to survive and resist common antibiotic substances. The most common germ is Methicillin-resistant Staphylococcus aureus (MRSA), which became an epidemic across the world in the 1990s, and still remains one of the difficult-to-treat ESKAPE pathogens (Enterococcus faecium, S. aureus, Klebsiella pneumoniae, Acinetobacter baumannii, Pseudomonas aeruginosa, and Enterobacter species) [6]. Even though new medications have been approved and widely used, each year in the United States, at least 2.8 million people become infected with bacteria that are resistant to antibiotics, and at least 35,000 people die each year, as a direct result of these infections [2].

Therefore, the race is on to find new treatments for these infections, with researchers exploring new therapies and sources for antibiotics. However, besides using antibiotics as directed and only when necessary, avoiding infections in the first place with appropriate immunization, safe food-handling practices and modern concepts of hygiene, should be our fist aim when treating patients.

\section{References}

1. Alduina R (2020) Antibiotics and environment. Antibiotics (Basel) 9(4):202

2. CDC (2019) Antibiotic resistance threats in the United States, 2019. U.S. Department of Health and Human Services CDC, Atlanta

3. Freiberg JA (2017) The mythos of laudable pus along with an explanation for its origin. J Commun Hosp Intern Med Perspect 7:196-198

4. Kadar N (2019) Rediscovering Ignaz Philipp Semmelweis (18181865). Am J Obstet Gynecol 220:26-39

5. Lister J (1870) Effects of the antiseptic system of treatment upon the salubrity of a surgical hospital. Lancet 1:40-42

6. Turner NA, Sharma-Kuinkel BK, Maskarinec SA, Eichenberger EM, Shah PP, Carugati M et al (2019) Methicillin-resistant Staphylococcus aureus: an overview of basic and clinical research. Nat Rev Microbiol 17:203-218

Publisher's Note Springer Nature remains neutral with regard to jurisdictional claims in published maps and institutional affiliations. 